\title{
Quantitative study of the immunoglobulin-containing cells in trephine samples of bone marrow
}

\author{
J CROCKER, RC CURRAN
}

From the Department of Pathology, The Medical School, Birmingham B15 2TJ

SUMMARY Immunoglobulin (Ig) was demonstrated in paraffin sections of 12 trephine bone marrow biopsies by means of the unlabelled antibody peroxidase-antiperoxidase (PAP) method. The Ig-

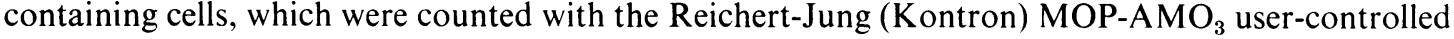
image-analyser, were found to constitute approximately $4.2 \%$ of all the nucleated cells in the marrow, a figure significantly higher than those reported by previous workers.

Estimates of the numbers of plasma cell numbers in bone marrow have generally been made on aspiration biopsies $^{12}$ and have been based on morphological criteria. One previous study made use of disaggregated aspirated marrow specimens with immunofluorescent demonstration of immunoglobulins. ${ }^{3}$ In the present study, plasma cells were detected by demonstrating immunoglobulin (Ig) within their cytoplasm by means of the unlabelled antibody peroxidase-antiperoxidase (PAP) method. Moreover, instead of aspiration biopsies, trephine samples were used. Eleven of the trephine samples were from patients being investigated for the extent of their malignancy and one was from a case of pyrexia of unknown origin. None showed histological evidence of neoplastic infiltration of the marrow and the haemopoietic tissue was reported as morphologically normal in all the specimens. Cell counting was greatly simplified by the use of the Reichert-Jung (Kontron) $\mathrm{MOP}-\mathrm{AMO}_{3}$ operator-controlled image analyser.

\section{Material and methods}

Twelve specimens of marrow were taken from the posterior iliac crest by means of a Jamshidi needle from the same number of patients. Six patients had nodal Hodgkin's disease, two had nodal nonHodgkin's disease, three had bronchial carcinoma and one had pyrexia of unknown origin.

FIXATION AND PROCESSING

Ten specimens were fixed for $1-5 \mathrm{~h}$ at $20^{\circ} \mathrm{C}$ in formol sublimate and two specimens for $24 \mathrm{~h}$ in formol

Accepted for publication 18 February 1981 saline containing $2 \%$ acetic acid. After this period of preliminary fixation, all specimens were fixed for two days at $20^{\circ} \mathrm{C}$ in formol saline solution to which $10 \%$ glacial acetic acid had been added. The latter solution also acted as a decalcifying agent. ${ }^{4}$ After fixation the specimens were dehydrated throug graded ethanols, cleared in chloroform and em bedded in Ralwax (RA Lamb). The high polymes content of Ralwax makes it particularly suitable for trephine specimens. Sections were cut at $3 \mu \mathrm{m}$.

\section{PAP METHOD}

Immunoglobulins $(\gamma, \alpha$ and $\mu$ heavy chains; $\kappa$ and $\lambda$ light chains*) were demonstrated by the PAP method. ${ }^{5}$ Trypsinisation was not used, being unnecessary with acetic acid-containing fixatives. ${ }^{4}$ The optimal dilution of the specific antiserum against light or heavy chain was $1 / 500$ or $1 / 1000$.

\section{CONTROLS}

Sections were exposed to DAB-hydrogen peroxide alone; normal rabbit serum was substituted for the 을 specific antiserum to light or heavy chain; and a range of dilutions of IgG, IgA and IgM was added to each specific antiserum to block the reaction to each heavy chain.

\section{COUNTING PROCEDURE}

The Reichert-Jung (Kontron) $\mathrm{MOP}-\mathrm{AMO}_{3}$ imageanalyser employs a sensitive light-emitting pen to count (or measure the area or maximum diameter of) $\frac{C}{\mathbb{D}}$ objects seen superimposed on the pen-tip by means of a conventional camera lucida drawing tube. The $T$ information thus obtained is fed to the programmed

*Dakopatts 10-090, 10-MAT, 10-091, 10-9K 2, 10-9L2, 21090, Z113 (Mercia Brocades Ltd, Weybridge, Surrey). 1080 
microprocessor and stored on one of the 20 channels available in the machine. At least 1000 nucleated marrow cells were counted in each PAP preparation and stored on one channel. For each field examined, the number of heavy or light chain-containing cells counted was stored and summated on another channel. At the end of this procedure, the machine printed out the totals on the two channels and expressed the number of heavy or light chaincontaining cells as a percentage of the total nucleated cell count for the fields examined. The number of cells containing each heavy or light chain was estimated separately. In this way the total percentage of cells containing a heavy chain $(\gamma, \alpha$ and $\mu)$ and the total percentage of cells containing a light chain ( $\kappa$ or $\lambda$ ) were obtained. The ratio of the number of $\kappa$-containing cells to $\lambda$-containing cells was also calculated.

\section{Results}

Apart from the occasional megakaryocytes which gave a weak positive cytoplasmic reaction for Ig $(\gamma, \alpha, \mu, \kappa$ and $\lambda$ chains) and which were not included in the count, the Ig-containing cells appeared to be plasma cells of varying maturity. The number of such cells containing the various types of heavy or light chain are shown in the Figure, expressed as a

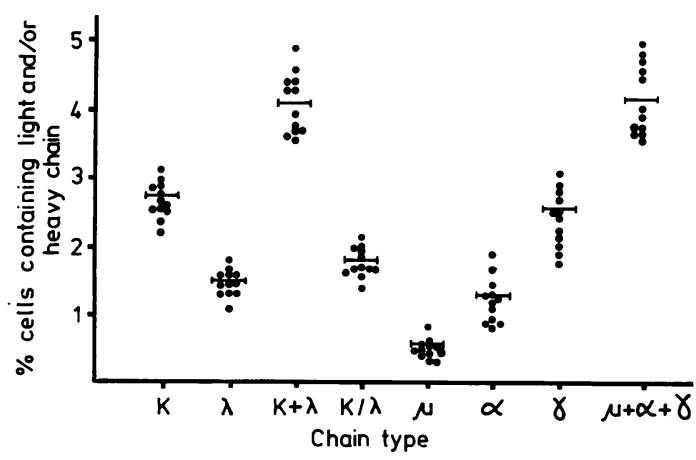

Number of cells containing various types of heavy or light chain as a percentage of total numbers of nucleated cells. Cross bars represent mean values. percentage of the total number of nucleated cells. The number of cells containing each type of chain is seen to vary only slightly from specimen to specimen. The Table shows the mean number of cells containing a heavy or light chain, expressed as a percentage of the total number of nucleated cells. The total percentage of cells containing heavy chains $(4 \cdot 17 \%)$ is close to the total percentage of cells containing light chains $(4 \cdot 18 \%)$, and the mean ratio of the $\kappa$ chain-containing cells to the number of $\lambda$ chaincontaining cells is $1 \cdot 78$. The Table also shows the number of cells containing a particular heavy chain $(\gamma, \alpha$ or $\mu)$ expressed as a percentage of all the cells containing a heavy chain ( $\gamma$ plus $\alpha$ plus $\mu$ ); thus, cells containing $\gamma$ chain constitute $56 \%$ of all heavy chain-containing cells, those with $\alpha$ chain $31 \%$, and with $\mu$ chain $13 \%$. Similarly, cells containing $\kappa$ chain form $64 \%$ of all light chain-containing cells, $\lambda$ chains being present in the other $36 \%$.

\section{Discussion}

Bone marrow trephine specimens are frequently taken for histological examination, as an adjunct to smear preparations, especially as part of a staging procedure for Hodgkin's disease and non-Hodgkin's lymphomas. Eleven of the 12 specimens used in the present study were taken as part of a staging procedure and all were reported as negative-that is, the haemopoietic tissue was histologically normal in all respects. The acetic acid-containing fixatives used effectively decalcified the sections and readily allowed the preparation of thin $(2-4 \mu \mathrm{m})$ flat paraffin sections which reacted well with the PAP method without prior trypsinisation. Cell morphology was also good, thereby facilitating the counting of cells on the Kontron MOP- $\mathrm{AMO}_{3}$ image analyser.

Quantification is being increasingly used in histopathology and automatic or semiautomatic measurements have been made on a variety of tissues, including lung, ${ }^{6}$ muscle ${ }^{7}$ and jejunal mucosa $;^{8}$ lymph node preparations have also been studied. ${ }^{9}$ The techniques now available are fast and accurate and the image analyser used in this study made the counting of large numbers of cells relatively easy.

Our finding of Ig in the cytoplasm of some mega-

Numbers of cells containing a heavy or light chain and the mean $\kappa: \lambda$ ratio

\begin{tabular}{|c|c|c|c|c|c|c|c|c|}
\hline & \multicolumn{5}{|c|}{$\%$ of cells containing: } & \multirow[t]{2}{*}{ Total $\gamma+a+!$} & \multirow[t]{2}{*}{ Total $\kappa+\lambda$} & \multirow[t]{2}{*}{ Ratio $\kappa: \lambda$} \\
\hline & $\gamma$ & $a$ & $\mu$ & $\kappa$ & $\lambda$ & & & \\
\hline Mean value & $\begin{array}{l}2 \cdot 34 \\
(56)\end{array}$ & $\begin{array}{l}1 \cdot 28 \\
(31)\end{array}$ & $\begin{array}{l}0.55 \\
(13)\end{array}$ & $\begin{array}{l}2.68 \\
(64)\end{array}$ & $\begin{array}{l}1.5 \\
(36)\end{array}$ & $4 \cdot 17$ & $4 \cdot 18$ & 1.78 \\
\hline Standard error of mean & $0 \cdot 12$ & 0.09 & 0.04 & 0.08 & 0.04 & $0 \cdot 13$ & $0 \cdot 10$ & 0.06 \\
\hline
\end{tabular}

Figures in brackets represent the \% of heavy and light chain-containing cells related to the total $\gamma, \alpha$ or $\mu$ chain-containing cells or $\kappa$ or $\lambda$ chaincontaining cells. 
karyocytes was unexpected. However, as with the plasma cells, the reaction was blocked by the addition of the appropriate heavy chain antigen to the specific antibody used in the first stage of the PAP sequence, confirming that Ig in the cytoplasm was responsible and not some contaminating antimegakaryocyte antibody in the reagent. Megakaryocytes do not synthesise Ig and presumably the Ig-positive cells absorbed immunoglobulin from the environment. Their presence did not interfere in any way with the counting of the Ig-containing cells of the plasma cell series.

In one published series, based on a morphological study of smears of bone marrow aspirates from 12 healthy men, plasma cells were found to constitute $1 \cdot 3 \%$ of all the nucleated marrow cells, ${ }^{1}$ and an upper limit of $2-3 \%$ is generally accepted. ${ }^{2}$ However, Turesson $^{3}$ used immunofluorescence to detect cells containing Ig in dispersed bone marrow preparations, obtaining a value of $3.01 \%$ for Ig-containing cells. The $\kappa: \lambda$ ratio was found to be 1.5 and the numbers of IgG-containing cells exceeded that for IgA-containing cells, with cells containing IgM being the smallest in number. Our figure for the $\kappa: \lambda$ ratio $(1 \cdot 78)$ is higher than that found by Turesson $(1 \cdot 5)$. However, our percentages of cells containing $\gamma, \alpha$ and $\mu$ heavy chains closely resemble Turesson's figures; whereas our data show $\gamma$ chain-containing cells to constitute $56 \%$ of the heavy chain-containing cells, $\alpha$ chain cells $31 \%$ and $\mu$ chain cells forming $13 \%$, in Turesson's series, $\gamma$-chain containing cells were $55 \%$ of cells containing heavy chains, $\alpha$ chain formed $30 \%$ and $\mu$ chain $15 \%$. We found Ig-containing cells to constitute a rather higher proportion of the nucleated cells in the marrow; thus the figure for $\kappa+\lambda$ light chain-containing cells was $4 \cdot 18 \%$ (range 3.73-4.90), and for $\gamma+\alpha+\mu$ heavy chain-containing cells it was $4 \cdot 17 \%$ (range $3 \cdot 60-4 \cdot 95$ ). The higher figures which we obtained for some cells may have resulted from the use of the sensitive PAP technique, better fixation or from the use of iliac crest trephines instead of sternal marrow aspirates. On the other hand it has been shown that the number of plasma cells is often increased in the bone marrow of patients with malignant disease. ${ }^{2}$ This could explain the larger numbers of Ig-containing cells in 11 of our preparations, but the fact that the total numbers of Ig-containing cells was more or less constant from patient to patient, irrespective of the type of malignancy, makes it unlikely. Certainly, if malignancy had any effect on the marrows, it must have been non-specific. However, the presence of malignant cells actually within the marrow might reasonably be expected to influence the number of Ig-containing cells there. We now propose to count the Ig-containing cells in marrows infiltrated by malignant cells, using the results of the present study as controls.

We are grateful to Mrs VE Adkins for typing the manuscript and to $\mathrm{Mr} \mathrm{J}$ Gregory for his expert technical assistance. Generous financial support was provided by The Royal Society and by the Endowment Fund Medical Research Committee of the Central Birmingham Health District.

\section{References}

${ }^{1}$ Wintrobe MM. Clinical haematology. Philadelphia: Lea and Febiger, 1974:62.

2 Rywlin AM. Histopathology of the bone marrow. Boston: Little, Brown and Company, 1976:77-8.

${ }^{3}$ Turesson I. Distribution of immunoglobulin-containing cells in human bone marrow and lymphoid tissue. Acta Med Scand 1976;199:293-304.

${ }^{4}$ Curran RC, Gregory J. The effects of fixation and processing on the immunohistochemical demonstration of immunoglobulin in paraffin sections of tonsil and bone marrow J Clin Pathol 1980;33:1047-57.

${ }^{5}$ Curran RC, Gregory J. Demonstration of immunoglobulin in cryostat and paraffin sections of human tonsil by immunofluorescence and immunoperoxidase techniques. J Clin Pathol 1978;31:974-83.

' Langston C, Thurlbeck WM. The use of simple image analysers in lung morphometry. $J$ Microsc $1978 ; 114: 89$ 99.

' Scott KWM, Hoy J. The cross sectional area of diaphragmatic muscle fibres in emphysema, measured by an automated image analysis system.JPathol 1976;120:121-8.

${ }^{8}$ Slavin G, Souter C, Robertson K, McDermott S, Paton K. Measurement in jejunal biopsies by computer-aided microscopy. J Clin Pathol 1980;33:254-61.

${ }^{9}$ Crocker J, Curran RC. A study of nuclear diameters in lymph node imprints using the Zeiss Microvideomat. $J$ Clin Pathol 1979;32:670-4.

Requests for reprints to: Dr J Crocker, Department of Pathology, The Medical School, Birmingham B15 2TJ, England. 